\title{
Childhood Acute Megakaryoblastic Leukemia
}

National Cancer Institute

\section{Source}

National Cancer Institute. Childhood Acute Megakaryoblastic Leukemia. NCI Thesaurus.

Code C7972.

An acute megakaryoblastic leukemia occurring in children. 